\title{
Aplicación del ejercicio físico como terapia en medicina del trabajo para pacientes con fibromialgia
}

\author{
Aplications of physical exercise as a therapy in occupational medicine in pa- \\ tients with fibromyalgia
}

\author{
Delfín Galiano Orea ', Borja Sañudo Corrales ${ }^{2}$
}

1. Ibermutuamur. MATEPPS n 274. Madrid. España.

2. Departamento de Educación Física. Facultad de Ciencias de la Educación. Universidad de Sevilla. Sevilla. España.

Recibido: 29-05-13

Aceptado: 02-07-13

\section{Correspondencia}

Delfín Galiano Orea

Ibermutuamur

Polig. Ind. Ctra. Amarilla

Rafael Beca Mateos, 7

41007 Sevilla (España)

Teléfono: 0034954532034

Fax: 0034954531924

Resumen

Objetivo: Determinar los principales parámetros cardio-respiratorios de las mujeres con fibromialgia y su clasificación en función del nivel de afectación para una posterior prescripción de actividad física.

Método: Una muestra de 32 mujeres (edad \pm sd; $53.3 \pm 6.6$ años) fueron clasificadas en dos grupos experimentales en función de su nivel de afectación y sintomatología; niveles moderado y alto. Ambos grupos se sometieron a dos pruebas de esfuerzo en tapiz rodante separadas una semana en el tiempo. La intensidad del ejercicio se incrementó hasta que los participantes alcanzasen su máxima capacidad $\left(\mathrm{VO}_{2 \max }\right)$. Los gases expirados, parámetros ventilatorios y frecuencia cardiaca (FC) se midieron continuamente durante el ejercicio y la valoración subjetiva del esfuerzo (RPE) controlada cada minuto en el test.

Resultados: Los valores medios de consumo de oxígeno pico $\left(\mathrm{VO}_{2 \text { pico }}\right)$ alcanzados por ambos grupos fueron $24.9 \pm 3.2 \mathrm{ml} \bullet \mathrm{kg}-1 \cdot \mathrm{min}-1$ (Gr1) frente a $21.5 \pm 2.1 \mathrm{ml} \bullet \mathrm{kg}-1 \cdot \mathrm{min}-1$ (Gr2), similares a otros estudios en mujeres con fibromialgia, aunque inferiores a otras poblaciones de referencia. En el resto de parámetros ventilatorios, aún siendo inferiores en el grupo con mayor nivel de afectación, no se muestran diferencias significativas.

Conclusiones: Hemos comprobado, como en función del nivel de afectación y la sintomatología de los pacientes con fibromialgia, sus capacidades cardiorrespiratorias son distintas; por tanto, no podremos prescribir un ejercicio con la misma intensidad a mujeres de ambos grupos. En base a estos resultados los profesionales podrán prescribir actividad física con mayor seguridad y control.

Med Segur Trab (Internet) 2013; 59 (232) 310-321

Palabras clave: mujeres, grupos de nivel, capacidad cardiovascular, actividad física, fibromialgia. Sintomatología. 


\section{Abstract}

Purpose: To determine groups in women with fibromyalgia syndrome (FM) based on symptomatology and determine cardiovascular parameters during treadmill exercise to prescribe them physical activity.

Methods: Women $(N=32$, age $=53.3 \pm 6.6 \mathrm{yr})$ were assigned into two different groups according to their functional capacity and symptomatology. During incremental treadmill exercise test, exercise intensity was increased until participants achieved volitional exhaustion $\left(\mathrm{VO}_{2 \max }\right)$. Expired respiratory gases, ventilator parameters and heart rate (HR) were measured continuously during exercise and RPE was assessed each minute during the test.

Results: Peak $\mathrm{VO}_{2}$ values for the group 1 were significantly different than for group $2(24,9 \pm 3,2$ $\mathrm{ml} \bullet \mathrm{kg}-1 \cdot \min -1$ (Group 1) and 21,5 $\pm 2,1 \mathrm{ml} \bullet \mathrm{kg}-1 \bullet \mathrm{min}-1$ (Group 2)) similar ones to the found in other studies with women with FM, altough lower than anther populations without this pathology. On the other hand, if we take into account the $\mathrm{VO}_{2 \mathrm{VT}}$, we found no significant differences between groups in both trials. We found also differences in all the evaluated parameters.

Conclusion: Depending on the level of affectation and the symptomatology of the FM patients, their aerobic capacities were differents; therefore, we are not be able to prescribe physical activity with the same intensity for both groups. According to those results, professionals could prescribe physical activity with a high security and control.

Med Segur Trab (Internet) 2013; 59 (232) 310-321

Key Words: women, cardiovascular capacities, level groups, physical activity, fibromyalgia. Symptoms. 


\section{INTRODUCCIÓN}

La fibromialgia (FM) es un síndrome muy común, ubicado respecto a su clasificación nosológica en la Reumatología, caracterizado por dolor musculo-esquelético crónico, rigidez, cansancio generalizado, perturbaciones del sueño y frecuentemente por alteraciones en su dimensión psicológica. En la actualidad no se conoce la etiología ni los mecanismos patogénicos precisos que actúan en ella; no obstante, las evidencias apuntan hacia un modelo integrador que comprendería diversas teorías entre las que destacaría una base genética, una disfunción del sistema nervioso autónomo o alteraciones en el procesamiento del dolor a nivel central y donde diversos mecanismos adicionales podrían estar envueltos.

En la población clínica, un estudio de la American College of Reumatology (ACR) ${ }^{1}$ diseñado para estudiar las necesidades de reumatólogos en EEUU, reveló que la FM suponía un $15,7 \%$ de las consultas reumatológicas, sólo superada por la artritis reumatoide (AR) con un $23,7 \%$. Estudios clínicos actuales establecen porcentajes de pacientes con FM entre el $10 \%$ y el $25 \%$, incluyendo los trabajos americanos referenciados de la ACR, España $^{2}$, Australia ${ }^{3}$, Canadá ${ }^{4}$, y Polonia e Israel $^{5}$. Esta patología se asigna al 4-20\% de los pacientes visitados, al 2-4\% de los visitados en atención primaria y al 7,5\% de los pacientes visitados en atención especializada ${ }^{6,7}$.

En nuestro país, donde mejor se reflejan estos aspectos es en el estudio de "Prevalencia e impacto de las enfermedades reumáticas en la población española" ${ }^{\text {, que }}$ ofrecen para la FM una prevalencia estimada en mayores de 20 años del 2,4\%. Por sexos, entre los hombres se estima en un $0,2 \%$, frente a un $4,2 \%$ en mujeres. En cuanto a la distribución en grupos de edad, la FM aparece en todas las edades, encontrándose un pico de prevalencia máxima (4,9\%) entre los 40 y 49 años ${ }^{9}$.

Según el estudio EPIDOR ${ }^{10}$, se estima que entre el 10 y el $20 \%$ de los pacientes que visitan las unidades de reumatología padecen FM, mientras que en servicios no especializados el porcentaje se establece entre el 2,1 y el 5,7\%.

Lo cierto es que esta patología puede acarrear una importante pérdida de calidad de vida (CDV) para el paciente y un menoscabo para todas sus actividades (trabajo, ocio, actividades de la vida diaria, etc.), lo que provoca importantes consecuencias en las relaciones laborales, sociales y familiares, utilización de servicios médicos y costes elevados por incapacidad laboral ${ }^{9,11}$. Aunque la situación varía según los países contemplen o no a la FM como motivo de incapacidad laboral permanente, algunos estudios informan que los pacientes afectados de FM poseen una peor función en sus capacidades físicas, pasan más días en cama y pierden más días de trabajo que los sujetos sanos ${ }^{12}$.

Mientras la literatura sobre epidemiología y tratamiento de la FM ha crecido considerablemente en los últimos años, hay poca información sobre su impacto económico. Como se ha indicado anteriormente, el enorme incremento de esta patología conlleva una importante repercusión psicosocial así como un enorme coste económico, ya que los pacientes con FM usan con mayor frecuencia los servicios de salud que otras poblaciones ${ }^{13}$. Para su análisis, estos costes suelen dividirse en costes directos, resultantes del uso de los servicios de salud y costes indirectos, resultantes de la reducción o cese de la actividad laboral como consecuencia de la enfermedad.

Varios estudios han estimado los costes médicos directos de la patología ${ }^{14}$. En ellos se muestra una media anual del coste en servicios de salud, incluyendo medicamentos, entre 1000 y $3500 €$. Los pacientes con FM tienen 2,6 veces más demandas médicas que la media de beneficiarios de los servicios de salud. Se ha estimado que los pacientes con FM acuden una media de diez veces al año a la consulta del médico ${ }^{14}$ y usan una media de tres fármacos diarios para el control del dolor ${ }^{15}$. A los altos costes que ocasiona la FM a la sanidad pública habría que añadir los correspondientes a aquellos costes generados con la incapacidad laboral que produce esta enfermedad. En un estudio realizado con 193 pacientes con FM, el 78,5\% de los que trabajaban estuvieron alguna vez de baja laboral a 
causa de la FM y el $6,7 \%$ recibían una prestación por incapacidad debida a dicha patología ${ }^{16}$. Las dos terceras partes de pacientes de FM trabajan y una cuarta parte ha recibido algún tipo de ayuda de la administración por incapacidad laboral ${ }^{17}$. En definitiva, a la alta prevalencia en minusvalías, se le une el coste de las indemnizaciones, lo que supone un elevado impacto económico entre los trabajadores, en quienes por cada dólar gastado en reclamaciones específicas de FM, la empresa gastaba de \$57 a \$143 ( 42 a $105 €)$ en costes directos o indirectos adicionales ${ }^{18}$. En Canadá se estima que este síndrome provoca un gasto sanitario público anual de 350 millones de dólares y un gasto de las aseguradoras privadas de 200 millones $^{7}$.

En nuestro país, el estudio EPISER ${ }^{8}$, también observó una asociación estadísticamente significativa entre presentar criterios de FM y la situación laboral del individuo. Estas personas estaban empleadas con menor frecuencia $(32,7 \%)$ y el $11,5 \%$ se encontraban en incapacidad laboral frente al 3,2\% de la población sin criterios de $\mathrm{FM}^{19}$. En un estudio longitudinal ${ }^{20}$ de cuatro años y medio se constató que se había casi triplicado la proporción de casos que estaban recibiendo algún tipo de prestación parcial o total (del 27\% al 63\%). La proporción de pacientes que seguía trabajando en dicho estudio se redujo de un $43 \%$ a un $38 \%$ al final del seguimiento.

La realidad es que la mayoría de los pacientes con FM son sedentarios y tienen una capacidad física por debajo de la media, situación que se acrecienta por el dolor, la fatiga o la depresión a las que deben hacer frente. Estas situaciones limitan las actividades de la vida diaria de los pacientes y afecta a numerosos aspectos de su CDV como su situación laboral o familiar. Sin embargo, aunque las opciones terapéuticas son múltiples, el tratamiento óptimo para la FM es aún desconocido debido a la heterogeneidad de los pacientes. Numerosas guías basadas en las evidencias científicas han evaluado un amplio rango de terapias tanto farmacológicas como no farmacológicas. Aunque dichos tratamientos continúan sin resolver de forma fiable las limitaciones funcionales y el deterioro en la CDV de estos pacientes, se ha sugerido que las intervenciones no farmacológicas tienen un efecto significativamente superior sobre la función que los fármacos ejercen en estos pacientes.

\section{Ejercicio físico en el manejo de la fibromialgia}

El ejercicio físico se ha mostrado como la principal estrategia no farmacológica en el manejo sintomático de estos pacientes; sin embargo, a pesar de ser reconocido como parte esencial en el control de la FM, no todos los aspectos clínicamente relevantes ni los elementos determinantes para su prescripción han sido identificados. Varias revisiones sistemáticas se propusieron dar respuesta a estas incógnitas, evaluando los efectos del ejercicio físico, incluyendo ejercicios aeróbicos, de fortalecimiento y/o para la mejora de la amplitud de movimiento, sobre el bienestar general, los principales síntomas y la función de sujetos con FM. Se pretendía determinar la efectividad de varios tipos de entrenamiento, diferentes volúmenes y cuál de las variables mejoraba en mayor medida por el ejercicio. De esta forma podrían establecerse guías adecuadas para la prescripción de actividad física en estos pacientes ${ }^{21-24}$.

Este tipo de programas tienen como objetivo principal evitar el círculo vicioso entre el dolor, los trastornos psicológicos y la inactividad, comunes en este síndrome ${ }^{25}$. Mientras que la fatiga subyacente, el dolor o la depresión pueden contribuir a estilos de vida sedentarios y la baja aptitud física ; varios estudios han demostrado que las mujeres con FM son capaces de realizar ejercicio aeróbico de intensidad moderada, ejercicios de fortalecimiento y flexibilidad ${ }^{24}$. Conseguir que los pacientes con FM inicien y mantengan un programa de ejercicio físico sigue siendo un reto.

Son numerosos los metaanálisis y revisiones sistemáticas que han recogido las recomendaciones de estudios con alta calidad metodológica para la prescripción de AF en personas con $\mathrm{FM}^{23,24}$. De estos estudios se pueden extraer evidencias sobre los beneficios a corto plazo de estos programas, que se han mostrado eficaces en el alivio del dolor $^{26-29}$, mejora la calidad del sueño ${ }^{30,31}$. Otorga numerosos beneficios en el bienestar 
psicológico ${ }^{27-29,32}$, como puede ser la mejora del estado de ánimo, el bienestar o la autoeficacia, importantes beneficios pueden esperarse, también, en la reducción de ansiedad $^{28,33}$, y la depresión ${ }^{26,28,33,34}$.

Como conclusión a estos estudios se pudo extraer una evidencia moderada de los beneficios que el ejercicio aeróbico tiene sobre el bienestar general, la función física y el dolor de estos pacientes. Sin embargo, las evidencias son limitadas respecto a los beneficios del entrenamiento de la fuerza muscular y de ejercicios de flexibilidad, si bien es cierto que no se han encontrado evidencias de que el ejercicio físico empeore las manifestaciones clínicas de la enfermedad ${ }^{21,23,24,35}$.

\section{Parámetros cardiovasculares y sintomatología en la prescripción de ejercicio físico}

El ejercicio es cada vez más usado en la rehabilitación de pacientes; sin embargo, para que este ejercicio sea eficaz es necesario que seamos cuidadosos en su prescripción y control. Esta intensidad debe ser suficiente para inducir efectos en el entrenamiento pero no tan alta como para ser perjudicial o poder incrementar los síntomas, reduciendo la adherencia de la propia paciente.

Como se ha comentado anteriormente, el ejercicio es uno de los componentes clave en el tratamiento de pacientes con FM, y la mayoría de los médicos recomiendan a los pacientes adoptar conductas más activas; el problema radica en cómo empezar un programa de actividad física y más aún como mantenerlo y supervisarlo una vez que haya empezado. Son muchos los facultativos sanitarios que se aventuran a prescribir ejercicio, pero no de forma individualizada y, obviamente, no en base a criterios objetivos ya que, para ello, sería necesario realizar una prueba de esfuerzo a cada paciente, siendo inviable en el ámbito laboral, aunque es posible en la actual estructura funcional de Ibermutuamur, la cual dispone de Unidades de Cardiología preparadas para ello, aunque tanto por medios económicos, como por el tiempo que requeriría este proceso, resulta dificultoso. Es por esto que hace necesario e imprescindible la utilización de una herramienta que nos permita no solo prescribir con ciertas garantías la intensidad del esfuerzo, sino también controlar la evolución de nuestros pacientes sin necesidad de dedicarles mucho tiempo y permitiendo, poco a poco, que este proceso se gestione de forma autónoma por cada sujeto.

Las recomendaciones de AF para el desarrollo y mantenimiento de la salud en adultos sugieren que los adultos (18-65 años) deben realizar un protocolo básico. Para mejorar la resistencia cardiorrespiratoria (entrenamiento aeróbico): (a) la frecuencia de ejercicio al menos 3 días por semana, (b) la intensidad de ejercicio suficiente para alcanzar o superar el $40 \%$ de la frecuencia cardiaca de reserva (rango $40 \%$ a $85 \%$ ) o el $64 \%$ de la frecuencia cardiaca máxima (rango 64\% a 94\%), (c) los períodos de sesiones de al menos 20 min de duración (rango 20 a $60 \mathrm{~min}$ ), ya sea con ejercicio continuo o intermitente en bloques de $10 \mathrm{~min}$, y el uso de cualquier modalidad de ejercicio aeróbico que impliquen el uso de los principales grupos de músculos en las actividades rítmicas, y (d) un período de tiempo total de al menos 6 semanas.

Para mejorar la fuerza muscular, los requisitos de ejercicio de dosificación fueron los siguientes: (a) frecuencia de 2 a 3 días por semana, y (b) un mínimo de un conjunto de 8 a 12 repeticiones a una intensidad que permita realizar entre 8 y 12 repeticiones de cada ejercicio, utilizando cualquier tipo de ejercicio de fortalecimiento. La dosificación para la flexibilidad fue: (a) la frecuencia de ejercicio $\geq 2$ días por semana, (b) la intensidad necesaria para causar un malestar leve, y (c) 3 a 4 repeticiones con una duración de 10 a 30 segundos.

Si bien estas son las recomendaciones para la población general, un creciente cuerpo de investigación evaluando los diferentes tipos de ejercicio en personas con FM ha demostrado la necesidad de establecer consideraciones especiales para este colectivo, que vendrían dadas por su sintomatología o el propio nivel inicial de sus capacidades ${ }^{36}$. 
Es necesario que se individualice la intensidad, duración y frecuencia de cada sesión según sea la capacidad de los pacientes; sin embargo, encontramos un grave problema en la prescripción de AF en FM. A modo de ejemplo, dos mujeres con FM, con la misma edad, tendrían teóricamente la misma frecuencia cardiaca máxima siguiendo la tradicional prescripción en base a la fórmula "220 - edad" que promulgan numerosos autores.

Este hecho puede conllevar que mujeres con diferentes niveles de afectación reciban un mismo programa de ejercicio repercutiendo negativamente en su condición. Abordar estas cuestiones ayudará a los profesionales para el diseño óptimo de programas terapéuticos adecuados para este grupo poblacional.

Los pacientes con FM tienen distintos niveles iniciales en sus capacidades físicas; algunos de ellos pueden ejercitarse a moderada-alta intensidad, mientras que para otros esa intensidad puede incrementar el dolor ${ }^{32}$. Tal y como se había reflejado, la intensidad en pacientes con dolor crónico no es constante, fundamentalmente en aquellos con FM cuyos síntomas son muy variables ${ }^{37}$, lo que plantea la necesidad de valorar la capacidad individual antes de iniciar cualquier programa con objeto de ajustar la intensidad del ejercicio.

En el ámbito de las MATEPSS, la fibromialgia es una de las patologías que con mayor frecuencia incrementan el gasto por incapacidad, sin que en muchas ocasiones podamos manejar dicho tiempo de baja laboral asociado con la eficacia terapéutica. Los consejos para dichas trabajadoras deberían estar fisiológicamente sustentados en argumentos basados en la evidencia científica, sobre todo en esta patología cuya "fisiopatología" es un reto para el colectivo médico. Podremos manejar adecuadamente la terapia farmacológica, e incluso la terapia cognitivo-conductual, pero no estaremos suficientemente acertados en el enfoque indispensable del ejercicio si no encontramos la relación entre grado de afectación y posibilidades reales de las pacientes. Si como se ha expresado con anterioridad, la literatura científica establece que el ejercicio físico es el elemento sustancial del manejo de la enfermedad, deberemos prescribir adecuadamente los protocolos de actividad, personalizando el programa a la situación fisiológica de la trabajadora.

Por otra parte resulta indispensable que a través de estos programas físicos se consigan los beneficios esperados, evitando el abandono, pues para el médico de control del proceso, el paso siguiente al consejo del ejercicio para el paciente, será el de establecer la similitud de la actividad física desarrollada como terapia con la actividad laboral habitual de la trabajadora, aspecto esencial para la incorporación al medio laboral y por tanto para la reducción de la IT. Todo ello requiere identificar la relación entre el grado de afectación y las posibilidades funcionales de la paciente, objetivo principal del presente estudio.

\section{MATERIAL Y MÉTODO}

\section{Participantes}

Formación de grupos no aleatoria. Dos grupos naturales (Gr1; n=16 y Gr2; n=16) entre 44-65 años ( $\mathrm{Gr} 1=52,7 \pm 6.0$ años; $\mathrm{Gr2}=55,4 \pm 7,2$ años) pertenecientes a un colectivo de trabajadoras del Aljarafe sevillano participaron de forma voluntaria en el estudio. Mantuvimos el criterio metodológico de nuestra línea de investigación iniciada en 2010. Durante los meses de marzo y abril de 2012 se determinaron los parámetros fisiológicos en ambos grupos previamente clasificados en función de su nivel de afectación como indica el Proceso Asistencial de fibromialgia (Consejería de Salud, 2005) ${ }^{38}$ usando una escala sintomática, número de "tender points" y el Fibromyalgia Impact Questionnaire (FIQ). Cada participante firmó previamente un consentimiento informado para realizar el estudio que cumplía con las normas éticas de la Declaración de Helsinki de 1975. Las características previas de ambos grupos se reflejan en la tabla I. 
Tabla I. Características de los sujetos en función del grupo experimental

\begin{tabular}{rcrrr}
\hline & \multicolumn{2}{c}{ Gr.1 } & \multicolumn{2}{c}{ Gr.2 } \\
\hline & Media & Sd & Media & Sd \\
\hline Edad & 54,12 & 5,94 & 55,38 & 7,37 \\
Peso & $68,95^{*}$ & 10,73 & 74,06 & 14,45 \\
Altura & 1,56 & 0,05 & 1,59 & 0,04 \\
IMC & 28,29 & 4,49 & 29,04 & 4,98 \\
Nivel AF & $2,81^{*}$ & 0,02 & 2,03 & 0,13 \\
Nivel Sintomático & $19,35^{*}$ & 5,47 & 27,38 & 4,76 \\
FIQ & $51,56^{*}$ & 12,46 & 70,21 & 5,75 \\
TP & $11,71^{*}$ & 4,61 & 14,88 & 3,36 \\
\hline
\end{tabular}

Gr.1: Nivel de afectación medio; Gr.2: Nivel de afectación grave.

AF: Nivel de actividad física. FIQ: Cuestionario de impacto de Fibromialgia.

TP: Puntos dolorosos.

* Diferencias significativas entre ambos grupos, $\mathrm{p}<0,05$.

\section{Protocolo de medida}

Para derivar a los sujetos a alguno de los dos grupos experimentales, se determinaron, el mismo día de la prueba, los niveles del FIQ en español ${ }^{39}$ en base a cuatro escalas analógicas visuales, correspondientes a las dimensiones del ámbito de la calidad de vida (física, psicológica y social), que constituyen el núcleo fundamental de la afectación en la FM. Se determinó el número de puntos dolorosos (TP) específicos de esta patología y una valoración del nivel sintomático. En base a los resultados obtenidos en los test, se definieron dos niveles de afectación:

- nivel de afectación medio (Grupo 1): aquellos que tenían una puntuación total en las tres pruebas menor a 60 puntos.

- nivel de afectación grave (Grupo 2): aquellos que tenían una puntuación global superior a 60 puntos.

Para aquellos sujetos que estuviesen en una zona intermedia ( \pm 5 puntos), se tuvo en cuenta además el nivel de actividad previa de los sujetos, en base a un cuestionario internacional de actividad física (IPAQ) y la historia clínica de los mismos junto con un cuestionario de salud (SF-36).

Una vez clasificados, los sujetos realizaron dos test máximos en tapiz rodante divididos aproximadamente una semana en el tiempo. Se usó el protocolo de Bruce modificado por Kaminsky (1993) ${ }^{40}$. Durante las pruebas se recogieron los gases espirados junto con la ventilación (VE), tasa respiratoria (RR) y coeficiente respiratorio (RQ) cada 5 segundos, por medio del MetaMax 3B (CORTEZ Biophysik GMBH, Germany) con tecnología "breath by breath". La frecuencia cardiaca se obtuvo telemétricamente a través de un pulsómetro (Polar; Seattle, WA).

Cada sujeto realizó ejercicios de calentamiento durante 5 minutos antes del test. Se determinaron escalones de 3 minutos y se pidió la valoración subjetiva del esfuerzo (RPE) cada minuto y el resto de parámetros, VE, RQ, FC y el consumo de oxígeno $\left(\mathrm{VO}_{2}\right)$ cada 5 segundos. Los criterios de maximalidad del test $\left(\mathrm{VO}_{2 \max }\right)$ fueron al menos dos de los siguientes: 1) $\mathrm{RQ}=1,0,2)$ Frecuencia Cardiaca Máxima=95\% del máximo estimado para su edad, y 3) Estabilidad de la curva del $\mathrm{VO}_{2}\left(2 \mathrm{ml} \cdot \mathrm{kg}^{-1} \cdot \mathrm{min}^{-1}\right)$ durante el último minuto del escalón de esfuerzo.

\section{Análisis estadístico}

Los análisis se desarrollaron usando el paquete estadístico SPSS 12.0 para Windows (SPSS Inc, Chicago, IL, USA). Se realizó un análisis descriptivo de los valores medios 
encontrados para cada variable y la validez de los datos se estimó usando la prueba de Pearson $\left(\mathrm{r}_{\mathrm{xy}}\right)$ entre ambos test con la $\mathrm{FC}$, consumo de oxígeno $\left(\% \mathrm{VO}_{2 \max }\right), \mathrm{RR}, \mathrm{VE}$ y RQ como variables dependientes.

\section{RESULTADOS}

32 mujeres cumplieron los criterios de inclusión del ACR y una vez clasificadas en función de su nivel de afectación, se obtuvieron medias de 11,7 \pm 4,6 TP en un nivel de afectación medio, frente a los 14,9 $\pm 3,4$ TP en mujeres con afectación grave, lo que supone unas puntuaciones significativamente superiores. Si valoramos las puntuaciones de FIQ y nivel sintomático, igualmente encontramos diferencias significativas entre ambos grupos (70,9 en el Grupo 1 frente a 97,6 en el Grupo 2).

Los valores medios de las variables de respuesta al ejercicio de los pacientes en ambos grupos se resumen en la Tabla II. El 63\% de los sujetos incluidos en el grupo 1 y tan solo el $40 \%$ del grupo 2 alcanzaron el $\mathrm{VO}_{2 \max }$ de acuerdo con nuestro criterio; sin embargo, todos los sujetos alcanzaron el umbral anaeróbico $\left(\mathrm{VO}_{2 \mathrm{VT}}\right)$.

Para el consumo de oxígeno pico $\left(\mathrm{VO}_{2 \text { pico }}\right)$ de los pacientes del grupo con menor nivel de afectación encontramos diferencias significativas respecto a los valores del grupo 2 en ambas pruebas $\left[26,2 \pm 3,6 \mathrm{ml} \cdot \mathrm{kg}^{-1} \cdot \mathrm{min}^{-1}(\mathrm{Gr} 1)\right.$ frente a $\left.22,1 \pm 2,5 \mathrm{ml} \cdot \mathrm{kg}^{-1} \cdot \mathrm{min}^{-1}(\mathrm{Gr} 2)\right]$ siendo estos valores ligeramente inferiores, aunque igualmente significativos, en la segunda prueba $\left[23,6 \pm 2,8 \mathrm{ml} \cdot \mathrm{kg}^{-1} \cdot \mathrm{min}^{-1}(\mathrm{Gr} 1)\right.$ frente a $\left.20,9 \pm 1,6 \mathrm{ml} \cdot \mathrm{kg}^{-1} \cdot \mathrm{min}^{-1}(\mathrm{Gr} 2)\right]$.

Otro hallazgo importante es que los valores de $\mathrm{FC}_{\max }, \mathrm{VE} \mathrm{y} \mathrm{RQ}$, fueron todos menores en el grupo con peor sintomatología al compararlo con el grupo de menor afectación. Respecto a la RPE, no hemos encontrado grandes diferencias entre los grupos de nivel, variando aproximadamente 1 punto entre ellos (13,6 $\pm 0,9$ frente a 14,2 $\pm 1,2$ en el grupo 2 ).

Tabla II. Parámetros fisiológicos y percepción del esfuerzo de los sujetos en ambas pruebas

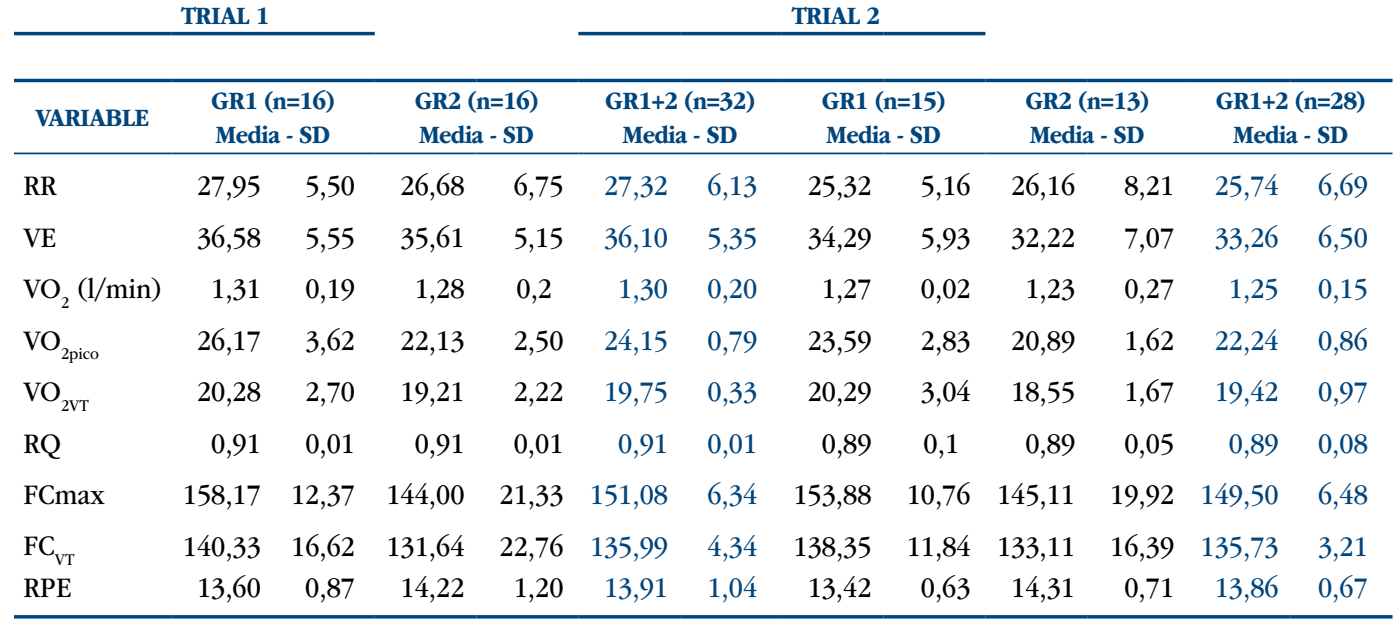

(Media $\pm S D) ; R R$ : Tasa respiratoria; VE: Ventilación; $\mathrm{VO}_{2}$ : Consumo de oxígeno; $\mathrm{VO}_{2 \text { ico }}$ : Máximo consumo de oxígeno alcanzado en el test; $\mathrm{VO}_{2 \mathrm{VT}}$ : Consumo de oxígeno en el umbral anaeróbico; RQ: Coeficiente de intercambio respiratorio; FCmax: Frecuencia cardiaca máxima; $\mathrm{FC}_{\mathrm{vT}}$ : Frecuencia cardiaca en el umbral anaeróbico; RPE: Valoración subjetiva del esfuerzo (Borg 6-20RPE).

\section{DISCUSIÓN}

Habitualmente muchos de los estudios fallan a la hora de no considerar grupos de nivel en fibromialgia, lo que desemboca en ocasiones en un tratamiento inadecuado ${ }^{41}$, 
por ello en esta muestra hemos comprobado como los sujetos del grupo 2 (con un mayor nivel de afectación) tienen un menor consumo de oxígeno y frecuencia cardiaca que sus compañeros del grupo 1 (nivel de afectación medio). Tan sólo 3 estudios han intentado evaluar previamente las capacidades cardio-respiratorias de los sujetos con FM comparando con sujetos control, aunque en ninguno se establecieron grupos de nivel ${ }^{19,42}$, confirmando el bajo nivel cardiorrespiratorio de los pacientes con FM al comparar con sujetos control sedentarios. En varios trabajos, por contra, se concluye diciendo que la capacidad aeróbica de estos pacientes parece ser normal al compararla con una población similar, aunque la percepción del esfuerzo a una intensidad relativa es mayor en mujeres con fibromialgia ${ }^{42}$. Respecto a la RPE media, los resultados no difieren mucho entre ambos grupos; sin embargo, al analizar estas diferencias por las medias de cada escalón de la prueba, encontramos diferencias cercanas a 2 puntos en la escala entre ambos grupos.

Numerosos autores consideran la capacidad aeróbica como uno de los factores determinantes en la patogénesis de la FM y hay evidencias de que al ejercitarse podemos modular el dolor en estos pacientes ${ }^{26,43}$. Aunque Bennett ${ }^{43}$ encontró que el $80 \%$ de los pacientes presentaba bajo nivel de condición física, Sietsema ${ }^{44}$ no encontró diferencias entre pacientes con FM y sujetos control usando para ello el $\mathrm{VO}_{2 \text { pico }} \mathrm{y} \mathrm{VO}_{2 \mathrm{VT}}{ }^{26}$. En nuestro estudio hemos encontrado diferencias significativas entre individuos con distinto nivel de afectación atendiendo a su $\mathrm{VO}_{2 \text { pico }}$.

Los valores obtenidos en este estudio, son algo inferiores a los reflejados por Meiworm ${ }^{45}$ aunque similares a los valores medios encontrados por otros autores ${ }^{43,44}$. Sin embargo, todos estos datos contrastan con los experimentados por nuestro grupo 2 , con mayor nivel de afectación $\left(22,1 \pm 2,5 \mathrm{ml} \cdot \mathrm{kg}^{-1} \cdot \mathrm{min}^{-1}\right)$.

Al comparar estos datos con los valores estándar en poblaciones sedentarias llama la atención como los valores en ambos grupos están muy por debajo de la media según los criterios de la ACSM para mujeres sedentarias entre 40 y 59 años ${ }^{46}$. Pudiendo compararse a los obtenidos por mujeres mayores de 70 años, ${ }^{47}$ o a los de mujeres con algún tipo de patología ${ }^{48}$. Por lo que respecta a nuestro grupo 2 , los resultados ponen de manifiesto la baja capacidad aeróbica de los mismos.

Numerosos autores han sugerido que los sujetos con fibromialgia deben ejercitarse para mejorar su capacidad aeróbica a una intensidad baja a moderada ${ }^{49,50}$, sin embargo, al no haber establecido subgrupos en su prescripción, la variabilidad entre sujetos de sus sesiones es amplia. En nuestro estudio al establecer grupos de nivel, tenemos una mayor seguridad en la prescripción ya que nuestros grupos son más homogéneos y sus capacidades físicas muy similares.

\section{CONCLUSIONES}

Tras la revisión de la literatura científica este es el primer estudio que analiza los parámetros cardiorrespiratorios de mujeres con fibromialgia clasificados en función de su sintomatología y nivel de afectación en población laboral. Estudios previos corresponden a la línea de investigación de nuestro grupo.

Los resultados muestran como el consumo de oxígeno es significativamente inferior en pacientes con mayor nivel de afectación, siendo éstos a su vez sensiblemente inferiores a los de poblaciones de la misma edad sin dicha patología, en consonancia con los estudios previos realizados por nosotros ${ }^{51}$.

El hecho de establecer grupos de nivel en la prescripción de ejercicio físico aeróbico en mujeres con fibromialgia nos permite reducir las diferencias individuales en cuanto a sus capacidades, asegurando así un mayor control sobre la intensidad de la sesión aconsejada.

Sugerimos una intensidad moderada para pacientes con un nivel de afectación medio y una intensidad baja para pacientes con un nivel de afectación grave o muy grave. 
Son necesarios otros estudios para entender los mecanismos mediante los cuales el ejercicio mejora los síntomas de las mujeres con fibromialgia y cual es la intensidad más adecuada para que dicho tratamiento sea más efectivo.

Resulta imprescindible incorporar en las asistencias de trabajadoras con incapacidad laboral temporal un protocolo de estratificación de su sintomatología al objeto de poder enfocar adecuadamente los programas de ejercicio físico que permitan la reincorporación laboral precoz.

\section{REFERENCIAS BIBLIOGRÁFICAS}

1. Marder, W.D., Meenan, R.F., y Felson, D.T. (1991). The present and future adequacy of rheumatology manpower. Arthritis Rheum, 34, 1204-1217.

2. Calabozo, R.M. (1998). Protocolo de actuación en fibromialgia. Protocolos de actuación en Reumatología. Burgos: Sociedad Española de Reumatología, 93-103.

3. Reilly, P.A., y Littlejohn, G.O. (1992). Peripheral arthralgic presentation of fibrositis/fibromyalgia syndrome. J Rheumatol, 19(2), 281-3.

4. White, K.P., Harth, M. y Teasell, R.W. (1995). Work Disability Evaluation and the Fibromyalgia Syndrome. Arthritis Rheum, 24(6), 371-381.

5. White, K.P., y Harth, M. (1998). The fibromyalgia problem. J Rheumatol, 25(5), 1022-1023.

6. Buskila, D., Neumann, L., Odes, L.R., Schleifer, E., Depsames, R., y Abu-Shakra, M. (2001). The prevalence of musculoskeletal pain and fibromyalgia in patients hospitalized on internal medicine wards. Sem Arthritis Rheum, 30, 411-417.

7. White, K.P., y Thompson, J. (2003). Fibromyalgia syndrome in an Amish community: a controlled study to determine disease and symptom prevalence. J Rheumatol, 30, 1835-40.

8. EPISER. (2001). Prevalencia e impacto de las enfermedades reumáticas en la población adulta española. Madrid: Sociedad Española de Reumatología.

9. Carmona, L., Ballina, J., Gabriel, R., Laffon, A. (2001). The burden of musculoeskeletal diseases in the general population of Spain: result from o nacional survey. Ann Rheum Dis, 60, 1040-5.

10. Gamero, F., Gabriel, R., Carbonell, J., Tornero, J., Sánchez-Magro, I. (2004). El dolor en las consultas de Reumatología españolas: estudio epidemiológico EPIDOR. Rev Clin Esp., 205(4):157-63.

11. Collado, A., Alijotas, J., Benito, P., Alegre, A., Romera, M., Sañudo, I., et al. (2002). Documento de consenso sobre el diagnóstico de la fibromialgia en Cataluña. Med Clin (Barc), 118, 745-9.

12. White, K.P., Nielson, W.R., Harth, M., Ostbye, T., y Speechley, M. (2002). Chronic widespread musculoskeletal pain with or without Wbromyalgia: psychological distress in a representative community adult sample. J Rheumatol, 29, 588-594.

13. Oliver, K., Cronan, T.A., Walen, H.R., y Tomita, M. (2001). Effects of social support and education on health care costs for patients with fibromyalgia. J Rheumatol, 28, 2711-9.

14. Penrod, J.R., Bernatsky, S., Adam, V., Baron, M., Dayan, N., y Dobkin, P.L. (2004). Health services costs and their determinants in women with fibromyalgia. J Rheumatol, 31(7), 1391-8.

15. Wolfe, F., Anderson, J., Harkness, D., Bennett, R.M., Caro, X.J., Goldenberg, D.L., Russell, I.J., y Yunus, M.B. (1997b). A prospective, longitudinal, multicenter study of service utilization and costs in fibromyalgia. Arthr Rheum, 40(9), 1560-1570.

16. Albornoz, J., Povedano, J., Quijada, J., De la Iglesia, J.L., Fernández, A., Pérez-Vilchez, D., et al. (1997). Características clínicas y sociolaborales de la fibromialgia en España: descripción de 193 pacientes. Rev Esp Reumatol, 24, 38-44.

17. Goldenberg, D.L. (1999). Fibromyalgia syndrome a decade later; what have we learned?. Arch Intern Med, 159(8), 777-785.

18. Robinson, R.L., Birnbaum, H.G., Morley, M.A., Sisitsky, T., Greenberg, P.E., y Claxton, A.J. (2003). Economic cost and epidemiological characteristics of patients with fibromyalgia. J Rheumatol, 30, 13181325.

19. Ubago, L., Ruiz, P., Bermejo, P., Olry, A., y Plazaola, C. (2005). Características clínicas y psicosociales de personas con fibromialgia. Repercusión del diagnóstico sobre sus actividades. Rev Esp Salud Pública, 79(6), 683-95.

20. Wigers, G.H., Stiles, T.C., y Vogel, P.A. (1996). Effects of aerobic exercise versus stress management treatment in fibromyalgia: a 4.5 year prospective study. Scand J Rheumatol, 25, 77-86. 
21. Rivera, J., Alegre, C., Ballina, F.J., Carbonell, J., Carmona, L., Castel, B., et al. (2006). Documento de consenso de la Sociedad Española de Reumatología sobre la fibromialgia. Reumatol Clin, 2(Supl 1), S55-66.

22. Brosseau, L., Wells, G.A., Tugwell, P., Egan, M., Wilson, K.G., Dubouloz, C.J., et al. (2008b). Ottawa Panel Members.Ottawa Panel evidence-based clinical practice guidelines for strengthening exercises in the management of fibromyalgia: part 2. Phys Ther, 88(7), 873-86.

23. Brosseau, L., Wells, G.A., Tugwell, P., Egan, M., Wilson, K.G., Dubouloz, C.J., et al. (2008a). Ottawa Panel Members.Ottawa Panel evidence-based clinical practice guidelines for aerobic fitness exercises in the management of fibromyalgia: part 1. Phys Ther, 88(7), 857-71.

24. Busch, A.J., Schachter, C.L., Overend, T.J., Peloso, P.M., y Barber, K.A. (2008). Exercise for fibromyalgia: a systematic review. J Rheumatol, 35(6), 1130-44.

25. Turk, D.C., Monarch, E.S., y Williams, A.D. (2002). Psychological evaluation of patients diagnosed with fibromyalgia syndrome: a comprehensive approach. Rheum Dis Clin North Am, 28, 219-33.

26. Valim, V., Oliveira, L., Suda, A., Silva, L., de Assis, M., Barros, T., Feldman, D., y Natour, J. (2003). Aerobic fitness effects in fibromyalgia. J Rheumatol, 30(5), 1060-9.

27. Da Costa, D., Abrahamowicz, M., Lowensteyn, I., Bernatsky, S., Dritsa, M., Fitzcharles, M.A., y Dobkin, P.L. (2005). A randomized clinical trial and individualized home-based exercise programme for women with fibromyalgia. Rheumatol (Oxford), 44, 1422-1427.

28. Tomas-Carus, P., Gusi, N., Häkkinen, A., Häkkinen, K., Leal, A., y Ortega-Alonso, A. (2008). Eight months of physical training in warm water improves physical and mental health in women with fibromyalgia: a randomized controlled trial. J Rehabil Med, 40(4), 248-52.

29. Sañudo B, Galiano D, Carrasco L, Bragojevic M, de Hoyo M, Saxton J. Aerobic exercise versus combined exercise therapy in women with fibromyalgia syndrome: a randomized controlled trial. Arch Phys Med Rehab. 2010; 91(12):1838-43.

30. Schachter, C.L., Busch, A.J., Peloso, P.M., y Sheppard, M.S. (2003). Effects of short versus long bouts of aerobic exercise in sedentary women with fibromyalgia: a randomized controlled trial. Phys Ther, 83, 340-358.

31. Valkeinen, H., Alen, M., Hannonen, P., Häkkinen, A., Airaksinen, O., y Häkkinen, K. (2004). Changes in knee extension and flexion force, EMG and functional capacity during strength training in older females with fibromyalgia and healthy controls. Rheumatology (Oxford), 43, 225-228.

32. Van Santen, M., Bolwijn, P., Landewe, R., Verstappen, F., Bakker, C., Hidding, A., et al. (2002b). High or low intensity aerobic fitness training in fibromyalgia: does it matter? J Rheumatol, 29, 582-587.

33. Bircan, C., Karasel, S.A., Akgün, B., El, O., y Alper, S. (2008). Effects of muscle strengthening versus aerobic exercise program in fibromyalgia. Rheumatol Int, 28(6), 527-32.

34. Assis, M.R., Silva, L.E., Alves, A.M., Pessanha, A.P., Valim, V., Feldman, D., et al. (2006) A randomized controlled trial of deep water running: clinical effectiveness of aquatic exercise to treat fibromyalgia. Arthritis Rheum, 55, 57-65.

35. Nishishinya, M.B., Rivera, J., Alegre, C., y Pereda, C.A. (2006). Intervenciones no farmacológicas y tratamientos alternativos en la fibromialgia. Med Clin (Barc), 127, 295-9.

36. Rooks, D.S. (2008). Talking to patients with fibromyalgia about physical activity and exercise. Curr Opin Rbeumatol, 20(2), 208-12.

37. Harris, R.E., Williams, D.A., McLean, S.A., Sen, A., Hufford, M., Gendreau, R.M., et al. (2005). Characterization and consequences of pain variability in individuals with fibromyalgia. Arthritis Rheum, 52(11), 3670-4.

38. Consejería de Salud. (2005). Fibromialgia: Proceso asistencial integrado. Sevilla: Junta de Andalucía.

39. Monterde S, Salvat I, Montull S, Ballart J. (2004). Validación de la versión española del Fibromyalgia Impact Questionnaire. Rev Esp Reumatol. 31(9):507-513.

40. Kaminsky LA, Roecker MS, Whaley MH, Dwyer GB. (1993). Evaluation of the BSU/Bruce ramp protocol. Med Sci Sports Exerc. 25:S13.

41. Thieme K, Spies C, Sinha P, Turk D, Flor H. (2005). Predictors of Pain Behaviors in Fibromyalgia Syndrome. Arthritis Care Res. 53(3):343-350.

42. Nielens H, Boisset V, Masquelier E. (2000). Fitness and Perceived Exertion in Patients With Fibromyalgia Syndrome. Clin J Pain. 16(3):209-13.

43. Bennett RM, Clark SR, Goldberg L. (1989). Aerobic fitness in patients with fibromyalgia-a controlled study of respiratory gas exchange and 133xenon clearance from exercising muscle. Arthritis Rheum. 32:454-60.

44. Sietsema KE, Cooper DM, Caro X, Leibling MR, Louie JS. (1993). Oxygen uptake during exercise in patients with primary fibromyalgia syndrome. J Rbeumatol. 20(5): 860-5. 
45. Meiworm L, Jakob E, Walker UA, Peter HH. (2000). Patients with flbromyalgia benefit from aerobic endurance exercise. Clin Rheumatol. 19:253-257.

46. American College of Sports Medicine. American College of Sports Medicine position stand. (1998). The recommended quantity and quality of exercise for developing and maintaining cardiorespiratory and muscular fitness, and flexibility in healthy adults. Med. Sci Sports and Exerc. 30:975-91.

47. Sanada K, Kuchiki TM, Miyachi MK, McGrath K, Higuchi M, Ebashi H. (2007). Effects of age on ventilatory threshold and peak oxygen uptake normalised for regional skeletal muscle mass in Japanese men and women aged 20-80 years. Eur J Appl Physiol. 99:475-483

48. Koltyn KF, Arbogast RW. (1998). Perception of pain alter resistance exercise. Br J Sports Med. 32(1):20-24.

49. Mannerkorpi K. (2005).Exercise in fibromyalgia. Curr Opin Rheumatol. 17:190-194.

50. Busch A, Schachter CL, Peloso PM, Bombardier C. (2007). Ejercicios para el tratamiento del síndrome de fibromialgia. (Revisión Cochrane traducida). En: La Biblioteca Cochrane Plus, número 1. Oxford, Update Software Ltd. Disponible en: http://www.update-software.com. (Traducida de The Cochrane Library, Issue 1. Chichester, UK: John Wiley \& Sons, Ltd.)

51. Sañudo B, Galiano D (2009). Using cardiovascular parameters and symptom severity to prescribe physical activity in women with fibromyalgia. Clin Exp Rheumatol 2009: 27 (Suppl. 56): S62-S66.

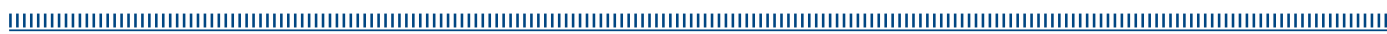

